\title{
Incipiently social carpenter bees (Xylocopa) host distinctive gut bacterial communities and display geographic structure as revealed by full-length $16 \mathrm{~S}$ sequencing
}

\author{
Madeline Handy ${ }^{1}$, Dino Sbardellati ${ }^{1}$, Michael $\mathrm{Yu}^{2}$, Nicholas Saleh ${ }^{3}$, Madeleine Ostwald $^{4}$, \\ and Rachel Vannette ${ }^{1}$ \\ ${ }^{1}$ University of California Davis \\ ${ }^{2}$ University of California Los Angeles \\ ${ }^{3}$ University of Florida Fort Lauderdale Research and Education Center \\ ${ }^{4}$ Arizona State University
}

February 2, 2022

\begin{abstract}
The gut microbiota of bees affect nutrition, immunity, and host fitness, yet the role of diet, sociality, and geographic variation in determining microbiome structure, including strain-level diversity and relatedness, remain poorly understood. Here, we use full-length $16 \mathrm{~S}$ amplicon sequencing to compare the crop and gut microbiomes of two incipiently social carpenter bee species, Xylocopa sonorina and Xylocopa tabaniformis, from multiple geographic sites within each species' range. We found that Xylocopa species share a set of core taxa consisting of Bombilactobacillus, Bombiscardovia, and Lactobacillus apis, found in $>95 \%$ of all individual bees sampled, and Gilliamella and Apibacter were also detected in the gut of both species with high frequency. The crop bacterial community of both species was comprised nearly entirely of Apilactobacillus with occasionally abundant nectar bacteria. Despite sharing core taxa, Xylocopa species' microbiomes were distinguished by multiple bacterial lineages, including species-specific strains of core taxa. In both bee species, bacterial species exhibited geographic patterns in the presence of specific sequence variants. The use of long-read amplicons revealed otherwise cryptic species and population-level differentiation in core microbiome members which was masked when a shorter fragment of the 16S (V4) was considered. We conclude that these Xylocopa species host a distinctive microbiome, similar to that of previously characterized social apids, which suggests that further investigation to understand the evolution of bee microbiome and its drivers is warranted.
\end{abstract}

\section{Introduction}

Gut microbial communities can be important mediators of host health and fitness (McFall-Ngai et al. 2013). Many bee species (Hymenoptera: Apoidea: Anthophila) host distinct and functionally important bacterial communities in the GI tract (Martinson et al. 2011, Lee et al. 2015, Moran 2015, Engel et al. 2016). As a lowdiversity and tractable experimental system, the bee gut represents an excellent model to examine metabolic specialization, function and coexistence within microbial communities (Engel et al. 2016). However, bee species vary in microbiome composition, including the presence of specialized taxa and the relative abundance of environmental bacteria (Kwong et al. 2017, McFrederick et al. 2017). The factors that predict this variation among species and their functions remain poorly understood (Engel et al. 2016), but sociality has been proposed as an important driver of gut microbiome evolution for bees, like other macroorganisms (Moeller et al. 2016, Kwong et al. 2017, Moran et al. 2019).

Social corbiculate bees in the subfamily Apinae ('pollen basket' bees), including honey bees and bumble bees, are characterized by distinctive gut microbial communities that are relatively consistent among individuals 
within a species (Kwong et al. 2017). In honey bees and bumble bees, gut bacterial communities are consistent among individuals and transmitted by social interactions (Koch and Schmid-Hempel 2011, Powell et al. 2014, Billiet et al. 2017). By contrast, in non-social bee species, including those closely related to social corbiculates (McFrederick and Rehan 2019), individuals host more variable and less distinctive microbiomes, likely driven by environmental rather than social acquisition of microbes (McFrederick et al. 2012, McFrederick and Rehan 2019, Cohen et al. 2020). However, key tests of the sociality hypothesis using bee species in the genus Halictus (which contains solitary and social species) found limited influence of sociality on bacterial composition (McFrederick et al. 2014, Rubin et al. 2018). These results raise the possibility that other traits instead of or in addition to sociality may be more important in shaping microbiome composition and specialization among bees.

Carpenter bee species in the genus Xylocopa (Apidae: Xylocopini) offer a unique system to study the relative role of sociality in structuring microbiome composition. Xylocopa are large-bodied bees and close phylogenetic relatives of social corbiculate apids (Bossert et al. 2019). Xylocopa are locally common and economically important pollinators in some systems (Keasar 2010, Giannini et al. 2015), and nest in timber or dead stalks of plants (Barrows 1980). Of particular note, several species of carpenter bees have been characterized as facultatively or incipiently social (Gerling et al. 1989, Michener 1990). In characterized species, the oldest female in a nest maintains reproductive dominance and feeds younger nestmates via trophallaxis (Lucia et al. 2015), but cooperative brood care is rarely documented. One species in which sociality has been wellstudied is $X$. sonorina, which lives in small, fluid, dominance-based societies with reproductive division of labor, where the proportion of individuals nesting socially is temporally dynamic (Ostwald et al. 2020). Moreover, in all of the best-studied Xylocopa species, both social and solitary nests are present within the same population (Gerling et al. 1989). Investigation of the microbiome of carpenter bees-close relatives of corbiculates with contrasting social structure- may offer insights into the role of sociality in the evolution of the bee microbiome.

Here, we examine the composition of bacterial full-length 16S rRNA genes in Xylocopa sonorina Smith [previously X. varipunctaPatton] and Xylocopa tabaniformis orpifex Smith (Bezark 2013), two carpenter bee species common in the western North America. We leverage PacBio Sequencing and a sample inference method with single-nucleotide resolution (Callahan et al. 2019) to examine strain-level resolution and phylogenetic relationships to previously characterized bee microbial taxa. These Xylocopa species often co-occur locally, collect nectar and pollen from the same plant hosts, and show a similar seasonal phenology in activity and reproduction. We examined microbiome composition in two tissues, the crop (foregut) and gut (combined midgut and hindgut), which are disparate in function and separated by a proventricular valve affecting movement between these regions. We hypothesized that the crop would be variable in bacterial composition among individuals, due to frequent intake of food including pollen, low microbial biomass and predominance of environmentally-sourced microbes, (Anderson et al. 2013). We hypothesized that if Xylocopa is similar to social apids, the gut would host a core microbiome distinctive from the crop that was consistent among individuals (Moran 2015), or if Xylocopamicrobiomes resemble solitary bees sampled to date, the gut would host a variable microbiome with high similarity to the crop (Voulgari-Kokota et al. 2019).

To address these hypotheses, we sampled bees from three geographic locations and first compared how bacterial alpha and beta-diversity differ among tissues, species and geographic locations. We also examined if sex or foraging status was associated with bacterial composition. Next, we defined the core bacterial taxa for these species and examined phylogenetic patterns among strains within these core clades. Lastly, to examine if shorter regions of the $16 \mathrm{~S}$ rRNA gene could also detect these patterns, we repeated analyses using the full-length data that had been trimmed to the V4 region only. Our results suggest that carpenter bees host distinctive gut bacterial communities including bacterial clades previously detected in corbiculates. In addition, Xylocopaspecies host phylogenetically and geographically distinct lineages within core clades which are revealed by the full-length $16 \mathrm{~S}$ but not by the V4 region alone.

\section{Materials and Methods}

\section{(A) Sample Collection}


Between 2019 and 2020, 33 X. sonorina and 22 X. tabaniformis adults were collected. Bees were captured in one of three ways: netted while foraging, caught using traps over the nest entrance (Supplementary Figure S1) or through excavation of logs to sample entire nests. The type of capture was recorded, with bees caught on the wing denoted as 'foraging' and those captured within or exiting a log denoted as 'nestcaught'. Samples were obtained from Davis, CA (21 X. sonorina; 14 X. tabaniformis ), Anza-Borrego Desert State Park in Southern California (1 X. sonorina , 8 X. tabaniformis ), and Tempe, Arizona (11 $X$. sonorina ). Samples from Davis and Anza-Borrego were captured during late summer and early fall while samples from Tempe were collected in early summer (see data file for collection date). Bee species can be distinguished morphologically, and identifications were verified using voucher specimens at the Bohart Museum of Entomology. Captured carpenter bees were photographed, then killed by placing them in a -20 ${ }^{\circ} \mathrm{C}$ freezer where they were stored until dissection.

\section{(B) Sample processing and DNA extraction}

Before dissection, carpenter bee samples were rinsed in $70 \%$ ethanol for fifteen seconds to minimize environmental microbe contamination. They were then air dried and placed in a sterile petri plate for dissection. Dissecting tools were flame sterilized before dissection and before each organ removal. The crop and the rest of the gut (combined midgut and hindgut) were separated and stored at $-20^{\circ} \mathrm{C}$ until DNA extraction.

Microbial DNA was extracted from crop and gut samples, extraction liquid, and kit reagents for an extraction control, using a modified procedure for the Qiagen DNeasy PowerSoil Kit (Rubin et al. 2014). Modifications include adding 4 magnetic beads per PowerBead Tube after tissue samples had been added and beating tubes in a BeadBlaster 24 Homogenizer for 3 cycles of speed 7 for 20 seconds per cycle. $60 \mu \mathrm{L}$ of Solution C1 and $2 \mu \mathrm{L}$ Proteinase K solution (600mAU/ML - from Qiagen Tissue and Blood) were then added to each tube and tubes were incubated overnight at $56^{\circ} \mathrm{C}$. The following day tubes were beaten once more using the same cycle settings and the rest of the protocol followed the manufacturer's protocol beginning at step 6. Briefly, full-length 16S region was amplified using 27F (Paliy et al. 2009) and 1492R (Lane 1991) and sequenced using PacBio Sequel v3 chemistry by the Dalhousie University Integrated Microbiome Resource facility (Halifax, Canada).

\section{(C) Bioinformatics}

Preliminary processing and filtering of raw full-length 16S rRNA reads into Amplicon Sequence Variants (ASVs) was performed in R v4.1.0 (R Core Team, 2021) using DADA2 (v1.20.0) (Callahan et al. 2019). Primers (F27 = AGRGTTYGATYMTGGCTCAG; R1492 = AAGTCGTAACAAGGTARCY) were removed, and reads were filtered by size and quality to yield sequences ranging from $1000-1600$ bp with no ambiguous bases, 2 maximum expected errors, and a minimum quality score of 3 . Filtered reads were then dereplicated, and sequencing errors were inferred using the PacBioErrfun function and removed. Chimeras were inferred with a minFoldParentOverAbundance value of 3.5 and removed using sequence consensus as a method. Finally, taxonomy was assigned using the BEExact database (Daisley and Reid 2021) and SILVA v 138.1 (Quast et al. 2012); resulting taxonomy was nearly identical (Supplementary File 1) and we present assignments from BEExact below.

The ASV and taxonomy tables generated from the DADA2 pipeline outlined above were merged with metadata using phyloseq (McMurdie and Holmes 2013). ASVs classified as chloroplast or mitochondria were removed and only samples with greater than 500 total sequences, where sampling curves saturated, were retained (Supplementary Figure S2). The extraction control contained only a single sequence and was removed at this step.

\section{(D) Statistical analysis}

\section{Alpha and beta diversity}

All statistical analyses were performed using R v.4.1.0. We examined rarefaction curves to assess if sampling depth was adequate and all samples plateaued (Supplementary Figure S2). Alpha diversity was quantified using Chao1 as an estimate of species richness, and Shannon diversity which also accounts for evenness of 
ASVs in a sample. To determine if host species, tissue type, their interactions, or sex and foraging type significantly affect diversity, a linear mixed effect model was constructed using lmer (Bates et al. 2011), with separate models for Chao1 and Shannon diversity. In both models, individual bee was included as a random effect and p-values were calculated using the lmerTest package (Kuznetsova et al. 2017).

To examine drivers of species composition among samples, we first examined if predictor variables, including species, tissue, geographic location, or sex contributed significantly to differences in species composition, using permutational ANOVA (PERMANOVA) using adonis2and assessed variation in dispersion among groups usingbetadisper (Oksanen et al. 2012). We present results using Bray-Curtis dissimilarities in the main text, but results are nearly identical to those based on weighted Unifrac dissimilarities (both presented in Supplementary Table S1). Differences between GI tract tissues were confirmed using PERMANOVA on a subset of the data containing only bees from which both the crop and gut sequenced successfully.

Because not all species of bee were sampled at each site, we conducted additional analyses to validate detected species and geographic effects in our dataset using stratified PERMANOVA. First, we subset the dataset to only samples collected in Davis, where both bee species were sampled and examined effects of bee species. Second, we examined the effect of sampling location separately for each bee species. In both cases, we used PERMANOVA implemented in adonis2 .

Additionally, to examine if bees caught foraging or nesting differed in bacterial diversity or composition, we repeated all alpha and beta diversity analyses on only $X$. sonorina samples, the only bee species for which we had nesting and foraging individuals. Models included sampling location, tissue, behavior and sex.

Assessing bacteria that distinguish bee species and are core to Xylocopa

To identify bacterial taxa that differ between host species and tissue type, we compared relative abundance of ASVs between bee species and tissues using DESeq2 (Love et al. 2013), with a false discovery rate FDR $<0.05$.

To identify if a core bacterial community was shared between the guts of X. sonorina and X. tabaninformis bees, individual ASVs were first collapsed at the genus_species level usingtax_glom(). The function eulerr::core_members() was then used to identify genus_species bins present at [?] $0.01 \%$ relative abundance in at least $95 \%$ of gut samples and heatmaps constructed to visualize the number of shared core taxa.

\section{Phylogenetic tree construction}

Within the core taxa identified above, we constructed phylogenies to compare our sequences to previously characterized bacteria from bees or other related habitats. Previously published $16 \mathrm{~S}$ rDNA sequences were downloaded from NCBI's 16S rRNA RefSeq database. Our strain list was comprised of previous studies of solitary and social bee-associated bacteria with published full-length16S sequences or genomes, as well as related strains within these groups (Lugli et al. 2017, Zheng et al. 2020).

To build trees, sequences were first alignied using AlignSeqs(Wright 2015). Pairwise distances of aligned sequences were calculated and used in the construction of neighbor joining trees optimized using internal maximum likelihood estimation (Schliep 2011) and visualized using ggtree (Yu et al. 2017).

\section{Assessing differences between full-length and $V_{4}$ datasets}

To generate a short-read dataset from our full-length sequences, we repeated the DADA2 pipeline but used V4 universal bacterial primers (V4_F = GTGCCAGCMGCCGCGGTAA V4_R = GGACTACHVGGGTWTCTAAT ) in place of PacBio sequencing primers, then repeated all downstream bioinformatics steps. To compare inference between datasets, we produced new phylogenetic trees and repeated our beta diversity analyses using this V4 dataset. The effects of different predictors on the beta diversity of our V4 dataset were assessed via adonis PERMANOVA tests, as with full-length sequences. In addition, to determine which V4 ASVs comprised which full-length ASVs, we mapped ASVs in the full-length dataset to their matching V4-only ASVs using the command bb.split.sh, part of the BBTools (Bushnell 2014) package. 


\section{Results}

\section{Sequencing results}

From the 110 bee samples submitted, 1 negative control, and 1 extraction liquid sample for PacBio long amplicon sequencing, 532,208 raw reads and 326,739 high-quality sequences were retained after processing. From these sequences, 810 unique amplicon sequence variants (ASVs) were inferred, 86 of which were classified as chloroplast or mitochondria and removed. The samples containing fewer than 500 total sequences (17, all crop and extraction or negative controls) were removed. Overall, the pipeline resulted in a final dataset containing 724 ASVs and 93 samples (55 gut and 38 crop) with a median of 2,472 sequences per sample. Sampling curves (Supplementary Figure S2) were saturating, indicating sufficient sampling depth.

Drivers of alpha and beta-diversity in Xylocopa microbiomes

Xylocopa gut samples hosted $\sim 222 \%$ more ASVs and had greater evenness than did crop samples (Figure 1; Chao1 lmer $\mathrm{F}_{1,54}=148 \mathrm{p}<0.001$; Shannon lmer $\left.\mathrm{F}_{1,45}=171 \mathrm{p}<0.001\right)$ across bee species. Xylocopa sonorina hosted marginally greater species richness than $X$. tabaniformis $\left(\mathrm{F}_{1,56}=3.78 \mathrm{p}=0.057\right)$ and significantly greater diversity $\left(\mathrm{F}_{1,51}=15.9, \mathrm{p}<0.001\right)$, while the interaction of tissue and species was not significant (Chao1 $\mathrm{F}_{1,54}=0.12, \mathrm{p}=0.73$; Shannon $\mathrm{F}_{1,45}=0.056 \mathrm{p}=0.81$ ). Within $X$. sonorina, female bees had significantly greater species richness and diversity within the gut than male bees (Chao1 $\mathrm{p}=0.001$, Shannon $\mathrm{p}=0.04$; Supplementary Figure S3), but no difference was detected in X. sonorina between sexes in the crop (Chao1 p>0.05; Shannon $\mathrm{p}>0.05)$, nor between sexes in either tissue for X. tabaniformis $(\mathrm{p}>0.2)$.

Bacterial species composition varied with sampling location, tissue type, species, their interactions, as well as the sex of bees (Figure 1D; full model Bray-Curtis PERMANOVA p $<0.05$ for all, Supplementary Table S1, Supplementary Figure S3). Paired analyses conducted on bees where both the crop and gut sequenced confirmed that crop and gut communities differed in both bee species (Supplementary Table S2; X. sonorina Bray $\mathrm{F}=5.63, \mathrm{R}^{2}=0.098, \mathrm{p}<0.001 ; X$. tabaniformis Bray $\left.\mathrm{F}=3.87, \mathrm{R}^{2}=0.16, \mathrm{p}<0.001\right)$. The gut and crop communities differed in dispersion. Using Bray-Curtis dissimilarities, the crop samples were more variable than the gut for both species (betadisper X. sonorina, $\mathrm{p}<0.05 ;$ X. tabaniformis $\mathrm{p}<0.01$; Fig $1 \mathrm{~B}$; Supplementary Figure S4).

In our validation analysis, species differed in bacterial composition when samples from a single location where both species were sampled were considered (Davis), for both the crop (Supplementary Table S3, Bray $\mathrm{R}^{2}=0.10 \mathrm{~F}=2.56, \mathrm{p}=0.01$ ) and the gut tissues (Bray $\mathrm{R}^{2}=0.21 \mathrm{~F}=8.52, \mathrm{p}<0.001$ ).

Geographic variation within species

Collection location and its interaction with Tissue type were highly significant in the full model (Supplementary Table S1) but to account for unbalanced sampling and understand drivers of these interactions, we also compared the effect of location within each species and tissue type separately. For X. sonorina, samples collected in Davis and Tempe locations differed in species composition for both the crop and gut (Supplementary Table S4; Bray-Curtis crop $\mathrm{R}^{2}=0.16 \mathrm{~F}=4.47 \mathrm{p}<0.001$; gut $\mathrm{R}^{2}=0.093 \mathrm{~F}=3.10 \mathrm{p}$ $<0.001$ ). For $X$. tabaniformis, samples from Davis and ABDSP differed in species composition, but only significantly in the gut, but to a lesser extent in the crop (Bray-Curtis dissimilarity gut $\mathrm{R}^{2}=0.17 \mathrm{~F}=4.18$ $\mathrm{p}<0.001$, crop $\left.\mathrm{R}^{2}=0.15 \mathrm{~F}=1.58 \mathrm{p}=0.073\right)$.

Effects of foraging behavior on bacterial composition

Within $X$. sonorina (the only species in which we sampled bees from nests and while foraging), bees caught foraging had significantly greater richness compared to nest-caught bees $\left(\mathrm{F}_{1,49}=4.6, \mathrm{p}=0.04\right)$ and a trend towards higher diversity (Supplementary Figure $\mathrm{S} 3 ; \mathrm{F}_{1,26}=3.1, \mathrm{p}=0.08$ ). Bacterial composition differed between foraging bees and nest-caught bees (PERMANOVA $\mathrm{p}=0.007)$ and between male and female bees $(\mathrm{p}=0.04)$ as well as with location and tissue $(\mathrm{p}<0.001)$. No male bees were captured while foraging and no significant interaction between tissue and foraging type was observed $(\mathrm{p}=0.07)$. Foraging bees exhibited a lower distance to centroid compared to nest-caught bees (betadisper behavior $\mathrm{p}=0.016$ ) and male bees had 
lower distance to centroid compared to females (betadisper sex $\mathrm{p}=0.001$ ). Notably, the crop of nest-inhabiting bees was nearly entirely comprised by Apilactobacillus, while the crop of foragers varied among individuals, with some foraging bees containing high abundance of known nectar inhabitants includingAcinetobacter nectaris and Neokomagataea thailandica .

Bacterial ASVs that distinguish species and tissue types

In the crop, 15 ASVs differed significantly between X. sonorinaand X. tabaniformis, 13 of which were classified as Apilactobacillus (DESeq2 FDR $<0.05$, Supplementary Figure S5). In the gut, 59 ASVs differed between Xylocopaspecies, 15 of which were classified as Bifidobacteriaceae (mostlyBombiscardovia), 27 as Lactobacillaceae (mostlyBombilactobacillus), and 6 Orbaceae (Gilliamella) (DESeq2 FDR <0.05, Supplementary Figure S5).

In addition ASVs also differentiated the crop and gut in X. sonorina and X. tabaniformis (Supplementary Figure S6). The crop was enriched in Apilactobacillus (X. sonorina only) and another undefined genus within the Lactobacillaceae (both species). In contrast, the gut of both species was enriched in many ASVs including from the genera Entomomonas , Bombiscardovia ,Bifidobacterium, Apibacter, with additional genera differentially abundant depending on the bee species considered, with more taxa enriched in the gut within $X$. sonorina .

Core Xylocopa gut bacteria

We detected 25 genus-species bins present in the gut of bothXylocopa species (Figure 2). Of those 25 taxa,Bombiscardovia coagulans, Bombilactobacillus bombi, andLactobacillus bxid5692 (BEExact ID number) were highly abundant and prevalent (present in at least $\sim 98 \%$ of gut samples) and we refer to these groups as 'core taxa' hereafter. DespiteEntomomonas having the highest average abundance of all shared bacterial taxa (Figure 2), it was not detected in a subset of $X$. sonorina individuals. Gilliamella was detected in $69.1 \%$ of samples and had an average of $4.36 \%$ relative abundance across all gut samples; Apibacter ( $87.3 \%$ of samples, $10.5 \%$ relative abundance) and Apilactobacillus, which was more frequently detected in the gut of $X$. sonorina ( $54.5 \%$ of samples) compared to $X$. tabaniformis ( $13.6 \%$ samples).

Phylogenetic analysis: core taxa ASVs display a high degree of host specificity

Phylogenetic and strain-level analysis suggests that the majority of the ASVs comprising core gut bacteria are host-specific (Figure 3, Figure 4). In contrast only a few ASVs span host species and locations, including the most abundant Lactobacillus sequence (ASV9). Moreover, while some ASVs are found at multiple geographic sampling locations, some are highly location-specific, particularly inBombilactobacillus and Bombiscordovia.

Bombilactobacillus bombi ASVs grouped into two clades (Figure 3b). One contained a known B. bombi isolated from bumble bees and $B$. mellis, isolated from honey bees while the other clade was phylogenetically distinct, detected only in X. sonorina, and found at all locations (Figure 3b). Lactobacillus bxid5692 ASVs formed a clade that was distinct from previous L. apis and aside from the most abundant ASV, was found primarily in X. sonorina(Figure 3c). The Bifidobacteriaceae genus Bombiscardovia coagulans also grouped into two clades, one of which contained a known $B$. coagulans isolated from bumble bees; the other more closely related to the newly described B. xylocopae. We detected extensive strain-level diversity within Bombiscordovia (Figure 4). Sequence variants were exclusively restricted to a single species and were often location-specific.

Comparison between long and short amplicon reads

Amplicon length influenced bacterial diversity and ecological inference. When only V4 regions were considered, 174 unique ASVs were detected (636 fewer than the full-length $16 \mathrm{~S}$ dataset at this step), 43 of which were classified as chloroplast or mitochondria and removed. Finally, 17 samples containing fewer than 500 sequences were removed, resulting in a final V4 dataset containing 93 samples with a median of 4,076 sequences per sample. Reduced sequence length in the V4 region resulted in the clustering of sequence variants from the full-length dataset: ASVs that in the full-length dataset distinguished host species or geographic 
location were considered a single ASV in the V4 dataset (Supplementary Figure S7-S9). However, despite a lower number of sequence, beta diversity inference was quite similar when either full-length or V4 regions was considered, with interactions among sex and sampling location less pronounced in the V4 dataset (Supplementary Tables S1-S4).

In addition, V4-region phylogenies contain fewer ASVs, most of which are found at high abundance in multiple host bee species, masking species-level differentiation in strains revealed by the full-length $16 \mathrm{~S}$ region (Supplementary Figure S7, S8).

\section{Discussion}

Our results demonstrate that the Xylocopa species examined here, although incipiently social, host a microbiome that exhibits similarities to previously characterized social corbiculates in at least two ways. First, Xylocopa individuals host a specific and distinctive set of bacterial taxa found consistently in bees that span geographic sampling locations, sexes, and individuals displaying different behaviors (nest-caught vs. foraging), unlike most previously characterized solitary bees (Voulgari-Kokota et al. 2019). Second, many of the bacterial lineages detected at high abundance in the gut have been previously described in social corbiculates (Kwong et al. 2017). Below, we discuss the specific taxa found and the implications for the effect of sociality on the evolution of the bee microbiome.

The bacterial taxa that we describe here are comprised of primarily bee-associated taxa or those that associate with diverse insects, similar to the previously characterized Xylocopa tenuiscapa (Subta et al. 2020). Xylocopa gut samples containedBombilactobacillus (previously Lactobacillus Firm4),Bombiscardovia ( Bifidobacteriaceae), and Lactobacillus bxid5692 (similar to Lactobacillus Firm5) in nearly every individual, often with multiple co-occurring ASVs, and typically at high abundance, similar to previously characterized Apis andBombus species (Kwong et al. 2017). Bombilactobacillus andBombiscardovia are considered core symbionts of adult bumble bees (Killer et al. 2010, Kwong et al. 2017, Parmentier et al. 2018) and primarily transmitted via social contact (Billiet et al. 2017). In addition, the full-length Bombiscardovia sequences in Xylocopa are diverse and cluster with B. coagulansisolated from Bombus, or closely related to the newly describedBifidobacterium xylocopae and B. actinocoloniiforme from European Xylocopa sp; suggesting that this bacterial group has a larger host range than previously recognized (Kwong et al. 2017, Alberoni et al. 2019, Hammer et al. 2021). In Apis mellifera ,Bombilactobacillus and Bifidobacterium sp. colonize the hindgut and are implicated in saccharide breakdown and fermentation (Lee et al. 2015). Despite similarities to the A. mellifera andBombus microbiome, the Xylocopa microbiomes characterized here are also distinct in the consistent presence of Apibacter, which is found consistently in Asian honey bees (Kwong et al. 2018) but only sporadically in Bombus. In contrast to Bombus, Xylocopa appears to mostly lack Snodgrassella - we detected a single ASV in low abundance. Gilliamella was frequently detected, but differed between species: $X$. sonorinahosted Gilliamella more consistently and at higher abundance in than did X. tabaniformis (Figure 2). In addition, the gut of bothXylocopa species consistently contained Entomomonas at relatively high frequencies (Supplementary Figure 6), a bacterium with a highly reduced genome and limited metabolic capabilities which was previously described in a diverse group of insects including Diptera, Coleoptera, and other Hymenoptera, including ants andApis (Wang et al. 2020). The ecological role of this genus is not understood. In X. sonorina, the crop was highly dominated by Apilactobacillus (Supplementary Figures S5a; S6a),previously documented in solitary bees and in the crop of social bees (McFrederick et al. 2018). Apilactobacillus may dominate in the provisions of some bee species where they have been hypothesized to inhibit pathogen growth or prevent spoilage of stored pollen (Vásquez and Olofsson 2009, McFrederick et al. 2018, Kapheim et al. 2021).

Since Xylocopa are incipiently social rather than classically eusocial (Gerling et al. 1981), their distinctive microbiome raises questions about the mechanisms required for effective social transmission of the microbiome. Dominant Xylocopa females will feed newly emerged nestmates via trophallaxis, and allow consumption of the stored provision (Gerling et al. 1989, Ostwald et al. 2021, Vickruck and Richards 2021). In this way, Xylocopa exhibit behavioral similarities to Apis, which engage in trophallaxis, andBombus, which do not engage in trophallaxis yet feed from shared food resources and engage in coprophagy (Näpflin and Schmid- 
Hempel 2016). In addition, Xylocopa individuals migrate among nests, including those of non-kin (Ostwald et al. 2021, Vickruck and Richards 2021). We hypothesize that these behaviors, as well as the relatively long lifespan (1-2 years), and large body size of Xylocopa could help explain the maintenance of specialized microbial taxa. Differences between Xylocopa species in microbiome composition and richness also suggest areas for future study. Although we lack details on X. tabaniformis sociality, this species is likely incipiently social like X. sonorina (Breed 1976) yet differs in breeding systems: males patrol flowers for mating, while X. sonorina males host non-resource based territories (Marshall and Alcock 1981). This could explain the differences in sex-specific microbiomes between species. Additional information on social structure or nesting biology may inform reduced sequence variant richness and more variable crop community in $X$. tabaniformis compared to X. sonorina .

In previous work, the microbiome of halictid bees that nest socially, including those that exhibit trophallaxis and eusociality (Kapheim et al. 2016), did not differ substantially from those that nest non-socially (McFrederick et al. 2014, Rubin et al. 2018), and resembled solitary bee microbiomes (Voulgari-Kokota et al. 2019), which are primarily environmentally acquired (McFrederick et al. 2012, Kapheim et al. 2021). The primary difference between solitary and social halictids was the abundance of Sodalis, an insect endosymbiont (Rubin et al. 2018). Combined, these studies suggest that not only social behaviors but perhaps additional other biological differences may be required for the maintenance of a distinctive bee microbiome.

Both Xylocopa species displayed microbiome differences among collection locations, suggesting populationlevel differentiation in microbiome composition. Indeed, many ASVs of core taxa were location-specific (Supplementary Figures 7, 8, 9), while only a few dominant ASVs (e.g. Lactobacillus bxid5692 ASV 9) was detected at all locations in both bee species. Sampling location explained between $9-16 \%$ of variation in bacterial composition, more than previous studies examining geographic signatures in honey bees (Ge et al. 2021) stingless bees (Liu et al. 2021), and even some solitary Osmia (Rothman et al. 2020). Although long-read sequences likely enable us to detect such patterns (Supplementary Table 1), distinctive Xylocopa sociality and patterns of microbial transmission may also contribute to geographic structuring.

As expected, we found that restricting our analysis to the V4 region resulted in fewer taxa detected, a loss of phylogenetic resolution (Supplementary Figure S7), and the loss of genetic information that could distinguish bacterial taxa between host species and among geographic locations (Supplementary Table S4). Due to reduced sequence length, ASVs that previously distinguished species and locations were collapsed into a single ASV (Supplementary Figure S 9), yet species and geographic location could still largely be distinguished using V4 sequences only (Supplementary Table S1). This comparison suggests that first-generation sequencing may be able to detect drivers of microbial community composition, but fail to show the extent of strainlevel differentiation that exists among populations and species. However, we caution that our comparative approach does not account for realistic primer bias or sequencing bias (Quail et al. 2012, Tedersoo et al. 2018) and as a result, may overestimate the similarity of these regions and their ecological inference. Nevertheless our data support the conclusion that long reads enable enhanced ecological insights into the strain-level composition and evolution of the microbiome, suggesting that despite its greater cost, this approach may be warranted when strain-level information may differentiate populations or closely related species (Tedersoo et al. 2021).

Overall, our results provide evidence that the microbiomes of species with simple social groups can have characteristics typically associated with the more complex eusociality of the corbiculate bees. Further work will be necessary to determine the role that specific features of sociality, such as trophallaxis, play in shaping the microbiome in the earliest stages of social evolution, and uncover the functional consequences of a specialized microbiome.

\section{Acknowledgements}

We thank the UC Davis Arboretum, UC Anza Borrego Desert Research Center and Davis homeowners for allowing us access to collect Xylocopasamples, the Bohart Museum of Entomology for access to voucher specimens, and members of the Vannette lab for comments on previous versions of this manuscript. We 
acknowledge funding to MH from UC Davis Provost's Undergraduate Research Fellowship and the UC Natural Reserve System through the Field Science Fellowship, and to RLV from National Science Foundation DEB \#1929516.

Data Accessibility and Benefit-Sharing: All sequence data will be accessible upon acceptance on NCBI Short Read Archive (project number \#\#\#\#), and all metadata and code will be available via Dryad and Zenodo (accession number \#\#\#\#). ${ }^{* *}$ draft code and metadata submitted with the current manuscript draft.

\section{References}

R Core Team. R: A Language and Environment for Statistical Computing. R Foundation for Statistical Computing. 2014. [http://www.R-project.org/].

Alberoni, D., F. Gaggìa, L. Baffoni, M. M. Modesto, B. Biavati, and D. Di Gioia. 2019. Bifidobacterium xylocopae sp. nov. and Bifidobacterium aemilianum sp. nov., from the carpenter bee (Xylocopa violacea) digestive tract. Systematic and Applied Microbiology42:205-216.

Anderson, K. E., T. H. Sheehan, B. M. Mott, P. Maes, L. Snyder, M. R. Schwan, A. Walton, B. M. Jones, and V. Corby-Harris. 2013. Microbial ecology of the hive and pollination landscape: bacterial associates from floral nectar, the alimentary tract and stored food of honey bees (Apis mellifera). PLoS ONE 8 :e83125.

Barrows, E. M. 1980. Results of a survey of damage caused by the carpenter bee Xylocopa virginica (Hymenoptera: Anthophoridae). Proc. Entomol. Soc. Wash. 82 :44.

Bates, D., M. Maechler, and B. Bolker. 2011. lme4: Linear mixed-effects models using S4 classes.

Bezark, L. G. 2013. Field Guide to the Common Bees of California: Including Bees of the Western United States. The Pan-Pacific Entomologist 89 :266-266.

Billiet, A., I. Meeus, F. Van Nieuwerburgh, D. Deforce, F. Wäckers, and G. Smagghe. 2017. Colony contact contributes to the diversity of gut bacteria in bumblebees (Bombus terrestris). Insect science24 :270-277.

Bossert, S., E. A. Murray, E. A. B. Almeida, S. G. Brady, B. B. Blaimer, and B. N. Danforth. 2019. Combining transcriptomes and ultraconserved elements to illuminate the phylogeny of Apidae. Molecular Phylogenetics and Evolution 130 :121-131.

Breed, M. D. 1976. The Evolution of Social Behavior in Primitively Social Bees: A Multivariate Analysis. Evolution 30 :234-240.

Bushnell, B. 2014. BBMap: a fast, accurate, splice-aware aligner. Lawrence Berkeley National Lab.(LBNL), Berkeley, CA (United States).

Callahan, B. J., J. Wong, C. Heiner, S. Oh, C. M. Theriot, A. S. Gulati, S. K. McGill, and M. K. Dougherty. 2019. High-throughput amplicon sequencing of the full-length 16S rRNA gene with single-nucleotide resolution. Nucleic acids research 47 :e103-e103.

Cohen, H., Q. S. McFrederick, and S. M. Philpott. 2020. Environment shapes the microbiome of the blue orchard bee, Osmia lignaria. Microbial Ecology 80 :897-907.

Daisley, B. A., and G. Reid. 2021. BEExact: a Metataxonomic Database Tool for High-Resolution Inference of Bee-Associated Microbial Communities. Msystems 6 :e00082-00021.

Engel, P., W. K. Kwong, Q. McFrederick, K. E. Anderson, S. M. Barribeau, J. A. Chandler, R. S. Cornman, J. Dainat, J. R. De Miranda, and V. Doublet. 2016. The bee microbiome: impact on bee health and model for evolution and ecology of host-microbe interactions. MBio 7 .

Ge, Y., Z. Jing, Q. Diao, J.-Z. He, and Y.-J. Liu. 2021. Host Species and Geography Differentiate Honeybee Gut Bacterial Communities by Changing the Relative Contribution of Community Assembly Processes. MBio12 :e00751-00721. 
Gerling, D., P. D. Hurd Jr, and A. Hefetz. 1981. In-nest behavior of the carpenter bee, Xylocopa pubescens Spinola (Hymenoptera: Anthophoridae). Journal of the Kansas Entomological Society:209-218.

Gerling, D., H. Velthuis, and A. Hefetz. 1989. Bionomics of the large carpenter bees of the genus Xylocopa. Annual review of Entomology34:163-190.

Giannini, T., S. Boff, G. Cordeiro, E. Cartolano, A. Veiga, V. Imperatriz-Fonseca, and A. Saraiva. 2015. Crop pollinators in Brazil: a review of reported interactions. Apidologie 46 :209-223.

Hammer, T. J., E. Le, A. N. Martin, and N. A. Moran. 2021. The gut microbiota of bumblebees. Insectes Sociaux 68 :287-301.

Kapheim, K. M., T.-Y. Chan, A. Smith, W. T. Wcislo, and P. Nonacs. 2016. Ontogeny of division of labor in a facultatively eusocial sweat bee Megalopta genalis. Insectes Sociaux 63 :185-191.

Kapheim, K. M., M. M. Johnson, and M. Jolley. 2021. Composition and acquisition of the microbiome in solitary, ground-nesting alkali bees. Scientific reports $11: 2993$.

Keasar, T. 2010. Large carpenter bees as agricultural pollinators. Psyche 2010 .

Killer, J., J. Kopečný, J. Mrázek, J. Havlík, I. Koppová, O. Benada, V. Rada, and O. Kofroňová. 2010. Bombiscardovia coagulans gen. nov., sp. nov., a new member of the family Bifidobacteriaceae isolated from the digestive tract of bumblebees. Syst Appl Microbiol $33: 359-366$.

Koch, H., and P. Schmid-Hempel. 2011. Socially transmitted gut microbiota protect bumble bees against an intestinal parasite. Proceedings of the National Academy of Sciences108 :19288-19292.

Kuznetsova, A., P. B. Brockhoff, and R. H. Christensen. 2017. lmerTest package: tests in linear mixed effects models. Journal of Statistical Software $82: 1-26$.

Kwong, W. K., L. A. Medina, H. Koch, K.-W. Sing, E. J. Y. Soh, J. S. Ascher, R. Jaffé, and N. A. Moran. 2017. Dynamic microbiome evolution in social bees. Science Advances 3 :e1600513.

Kwong, W. K., M. I. Steele, and N. A. Moran. 2018. Genome Sequences of Apibacter spp., Gut Symbionts of Asian Honey Bees. Genome biology and evolution 10 :1174-1179.

Lane, D. J. 1991. 16S/23S rRNA sequencing. Pages 115-175 in E. Stackebrandt, and Goodfellow, M., editor. Nucleic acid techniques in bacterial systematics. John Wiley and Sons, New York, NY.

Lee, F. J., D. B. Rusch, F. J. Stewart, H. R. Mattila, and I. L. Newton. 2015. Saccharide breakdown and fermentation by the honey bee gut microbiome. Environmental microbiology $17: 796-815$.

Liu, H., M. A. Hall, L. E. Brettell, M. Halcroft, J. Wang, S. Nacko, R. Spooner-Hart, J. M. Cook, M. Riegler, and B. Singh. 2021. Gut microbial diversity in stingless bees is linked to host wing size and is influenced by geography. bioRxiv:2021.2007.2004.451070.

Love, M., S. Anders, and W. Huber. 2013. Differential analysis of count data, the DESeq2 package.

Lucia, M., V. H. Gonzalez, and A. H. Abrahamovich. 2015. Systematics and biology of Xylocopa subgenus Schonnherria (Hymenoptera, Apidae) in Argentina. ZooKeys:129.

Lugli, G. A., C. Milani, F. Turroni, S. Duranti, L. Mancabelli, M. Mangifesta, C. Ferrario, M. Modesto, P. Mattarelli, K. Jiř́, D. van Sinderen, and M. Ventura. 2017. Comparative genomic and phylogenomic analyses of the Bifidobacteriaceae family. BMC Genomics $18: 568$.

Marshall, L. D., and J. Alcock. 1981. The evolution of the mating system of the carpenter bee Xylocopa varipuncta (Hymenoptera: Anthophoridae). Journal of Zoology 193 :315-324.

Martinson, V. G., B. N. Danforth, R. L. Minckley, O. Rueppell, S. Tingek, and N. A. Moran. 2011. A simple and distinctive microbiota associated with honey bees and bumble bees. Molecular Ecology20 :619-628. 
McFall-Ngai, M., M. G. Hadfield, T. C. Bosch, H. V. Carey, T. Domazet-Lošo, A. E. Douglas, N. Dubilier, G. Eberl, T. Fukami, and S. F. Gilbert. 2013. Animals in a bacterial world, a new imperative for the life sciences. Proceedings of the National Academy of Sciences110 :3229-3236.

McFrederick, Q. S., and S. M. Rehan. 2019. Wild Bee Pollen Usage and Microbial Communities Co-vary Across Landscapes. Microbial Ecology77 :513-522.

McFrederick, Q. S., J. M. Thomas, J. L. Neff, H. Q. Vuong, K. A. Russell, A. R. Hale, and U. G. Mueller. 2017. Flowers and Wild Megachilid Bees Share Microbes. Microbial Ecology 73 :188-200.

McFrederick, Q. S., H. Q. Vuong, and J. A. Rothman. 2018. Lactobacillus micheneri sp. nov., Lactobacillus timberlakei sp. nov. and Lactobacillus quenuiae sp. nov., lactic acid bacteria isolated from wild bees and flowers. International Journal of Systematic and Evolutionary Microbiology 68 :1879-1884.

McFrederick, Q. S., W. T. Wcislo, M. C. Hout, and U. G. Mueller. 2014. Host species and developmental stage, but not host social structure, affects bacterial community structure in socially polymorphic bees. Fems Microbiology Ecology 88 :398-406.

McFrederick, Q. S., W. T. Wcislo, D. R. Taylor, H. D. Ishak, S. E. Dowd, and U. G. Mueller. 2012. Environment or kin: whence do bees obtain acidophilic bacteria? Molecular Ecology 21 :1754-1768.

McMurdie, P. J., and S. Holmes. 2013. phyloseq: an R package for reproducible interactive analysis and graphics of microbiome census data. Plos One 8 :e61217.

Michener, C. 1990. Castes in Xylocopine Bees. Pages 123-146 in E. W., editor. Social Insects: An Evolutionary Approach to Castes and Reproduction. Springer Verlag.

Moeller, A. H., S. Foerster, M. L. Wilson, A. E. Pusey, B. H. Hahn, and H. Ochman. 2016. Social behavior shapes the chimpanzee pan-microbiome. Science Advances 2 :e1500997.

Moran, N. A. 2015. Genomics of the honey bee microbiome. Current Opinion in Insect Science 10 :22-28.

Moran, N. A., H. Ochman, and T. J. Hammer. 2019. Evolutionary and ecological consequences of gut microbial communities. Annual Review of Ecology, Evolution, and Systematics 50 :451-475.

Näpflin, K., and P. Schmid-Hempel. 2016. Immune response and gut microbial community structure in bumblebees after microbiota transplants. Proceedings of the Royal Society B: Biological Sciences283 20160312.

Oksanen, J., F. G. Blanchet, R. Kindt, P. Legendre, R. B. O'Hara, G. L. Simpson, P. Solymos, M. H. Stevens, and H. Wagner. 2012. vegan: Community Ecology Package.

Ostwald, M., R. A. Dahan, Z. Shaffer, and J. H. Fewell. 2021. Fluid nest membership drives variable relatedness in groups of a facultatively social bee. Frontiers in Ecology and Evolution:893.

Ostwald, M., B. Lyman, Z. Shaffer, and J. Fewell. 2020. Temporal and spatial dynamics of carpenter bee sociality revealed by CT imaging. Insectes Sociaux $67: 203-212$.

Paliy, O., H. Kenche, F. Abernathy, and S. Michail. 2009. High-throughput quantitative analysis of the human intestinal microbiota with a phylogenetic microarray. Applied and environmental microbiology75 :3572-3579.

Parmentier, A., I. Meeus, F. Van Nieuwerburgh, D. Deforce, P. Vandamme, and G. Smagghe. 2018. A different gut microbial community between larvae and adults of a wild bumblebee nest (Bombus pascuorum). Insect science25 :66-74.

Powell, J. E., V. G. Martinson, K. Urban-Mead, and N. A. Moran. 2014. Routes of acquisition of the gut microbiota of the honey bee Apis mellifera. Applied and Environmental Microbiology 80 :7378-7387.

Quail, M. A., M. Smith, P. Coupland, T. D. Otto, S. R. Harris, T. R. Connor, A. Bertoni, H. P. Swerdlow, and Y. Gu. 2012. A tale of three next generation sequencing platforms: comparison of Ion Torrent, Pacific 
Biosciences and Illumina MiSeq sequencers. BMC genomics13 :1-13.

Quast, C., E. Pruesse, P. Yilmaz, J. Gerken, T. Schweer, P. Yarza, J. Peplies, and F. O. Glöckner. 2012. The SILVA ribosomal RNA gene database project: improved data processing and web-based tools. Nucleic acids research 41 :D590-D596.

Rothman, J. A., D. L. Cox-Foster, C. Andrikopoulos, and Q. S. McFrederick. 2020. Diet Breadth Affects Bacterial Identity but Not Diversity in the Pollen Provisions of Closely Related Polylectic and Oligolectic Bees. Insects $11: 645$.

Rubin, B. E., J. G. Sanders, J. Hampton-Marcell, S. M. Owens, J. A. Gilbert, and C. S. Moreau. 2014. DNA extraction protocols cause differences in 16S rRNA amplicon sequencing efficiency but not in community profile composition or structure. MicrobiologyOpen3 :910-921.

Rubin, B. E. R., J. G. Sanders, K. M. Turner, N. E. Pierce, and S. D. Kocher. 2018. Social behaviour in bees influences the abundance of Sodalis (Enterobacteriaceae) symbionts. Royal Society open science5 :180369-180369.

Schliep, K. P. 2011. phangorn: phylogenetic analysis in R. Bioinformatics 27 :592-593.

Subta, P., P. Yodsuwan, R. Yongsawas, A. In-on, N. Warrit, S. Panha, K. Khongphinitbunjong, P. Chantawannakul, K. Attasopa, and T. Disayathanoowat. 2020. Bacterial Communities in Three Parts of Intestinal Tracts of Carpenter Bees (Xylocopa tenuiscapa). Insects11 :497.

Tedersoo, L., M. Albertsen, S. Anslan, and B. Callahan. 2021. Perspectives and benefits of high-throughput long-read sequencing in microbial ecology. Applied and environmental microbiology 87 :e00626-00621.

Tedersoo, L., A. Tooming-Klunderud, and S. Anslan. 2018. PacBio metabarcoding of Fungi and other eukaryotes: errors, biases and perspectives. New Phytologist 217 :1370-1385.

Vasquez, A., and T. C. Olofsson. 2009. The lactic acid bacteria involved in the production of bee pollen and bee bread. Journal of apicultural research $48: 189-195$.

Vickruck, J. L., and M. H. Richards. 2021. Competition drives group formation and reduces within nest relatedness in a facultatively social carpenter bee. Frontiers in Ecology and Evolution:840.

Voulgari-Kokota, A., Q. S. McFrederick, I. Steffan-Dewenter, and A. Keller. 2019. Drivers, diversity, and functions of the solitary-bee microbiota. Trends in microbiology 27 :1034-1044.

Wang, J., Q. Su, X. Zhang, C. Li, S. Luo, X. Zhou, and H. Zheng. 2020. Entomomonas moraniae gen. nov., sp. nov., a member of the family Pseudomonadaceae isolated from Asian honey bee gut, possesses a highly reduced genome. International Journal of Systematic and Evolutionary Microbiology 70 :165-171.

Wright, E. S. 2015. DECIPHER: harnessing local sequence context to improve protein multiple sequence alignment. BMC bioinformatics16 :1-14.

Yu, G., D. K. Smith, H. Zhu, Y. Guan, and T. T. Y. Lam. 2017. ggtree: an R package for visualization and annotation of phylogenetic trees with their covariates and other associated data. Methods in Ecology and Evolution 8 :28-36.

Zheng, J., S. Wittouck, E. Salvetti, C. M. Franz, H. Harris, P. Mattarelli, P. W. O'Toole, B. Pot, P. Vandamme, and J. Walter. 2020. A taxonomic note on the genus Lactobacillus: Description of 23 novel genera, emended description of the genus Lactobacillus Beijerinck 1901, and union of Lactobacillaceae and Leuconostocaceae.

Figure legends

Figure 1. Microbiome comparison of two carpenter bee species A)Xylocopa sonorina and Xylocopa tabaniformis, photos by K. Garvey, used with permission. In B) bacterial species Chao 1 richness and Shannon's index diversity. In C), species and tissues differ in beta diversity, with the gut communities tightly clustering 
and differentiated between species. In D), bacterial composition in bothXylocopa species and tissue types is summarized at the Genus level.

Figure 2. Heatmap of bacterial genus-species bins detected in at least one sample of both Xylocopa species. Bacterial taxa are ordered by descending mean relative abundance in each bee gut sample.

Figure 3. Phylogenetic placement of gut Xylocopa core taxa in the Lactobacillaceae. A) Neighbor-joining phylogenetic tree optimized with internal maximum likelihood, where nodes with $>75 \%$ bootstrap support from 1000 resampled trees are colored blue, while those with $<75 \%$ bootstrap support at colored red. Core ASVs present at $>=0.1 \%$ relative abundance in at least one sample were used. Core taxa clades are indicated in green for Bombilactobacillus and blue forLactobacillus. Subtree in B) shows Bombilactobacillus (green tips in full Lactobacillaceae tree) ASVs from the current study (indicated as ASV) and their average relative abundance in individual bee gut tissue of $X$. sonorina and X. tabaniformis, with stacked bars colored by geographic location. Subtree in C) shows Lactobacillus ASVs (blue tip in full Lactobacillaceae tree) from the current study and their average relative abundance in individual bee gut tissue of $X$. sonorina and $X$. tabaniformis, with stacked bars colored by geographic location.

Figure 4. Phylogenetic placement of gut Xylocopa core taxa in the Bifidobacteriaceae, with outgroups selected from (Lugli et al. 2017). A) Neighbor-joining phylogenetic tree optimized with internal maximum likelihood, where nodes with $>75 \%$ bootstrap support from 1000 resampled trees are colored blue, while those with $<75 \%$ bootstrap support at colored red. Core ASVs present at $>=0.1 \%$ relative abundance in at least one sample were used. Core taxa clades are indicated in red for Bombiscardovia. Subtree in B) shows Bombiscardovia (red tips in full tree) ASVs from the current study (indicated as ASV) and their average relative abundance in individual bee gut tissue of $X$. sonorina and $X$. tabaniformis, with stacked bars colored by geographic location.

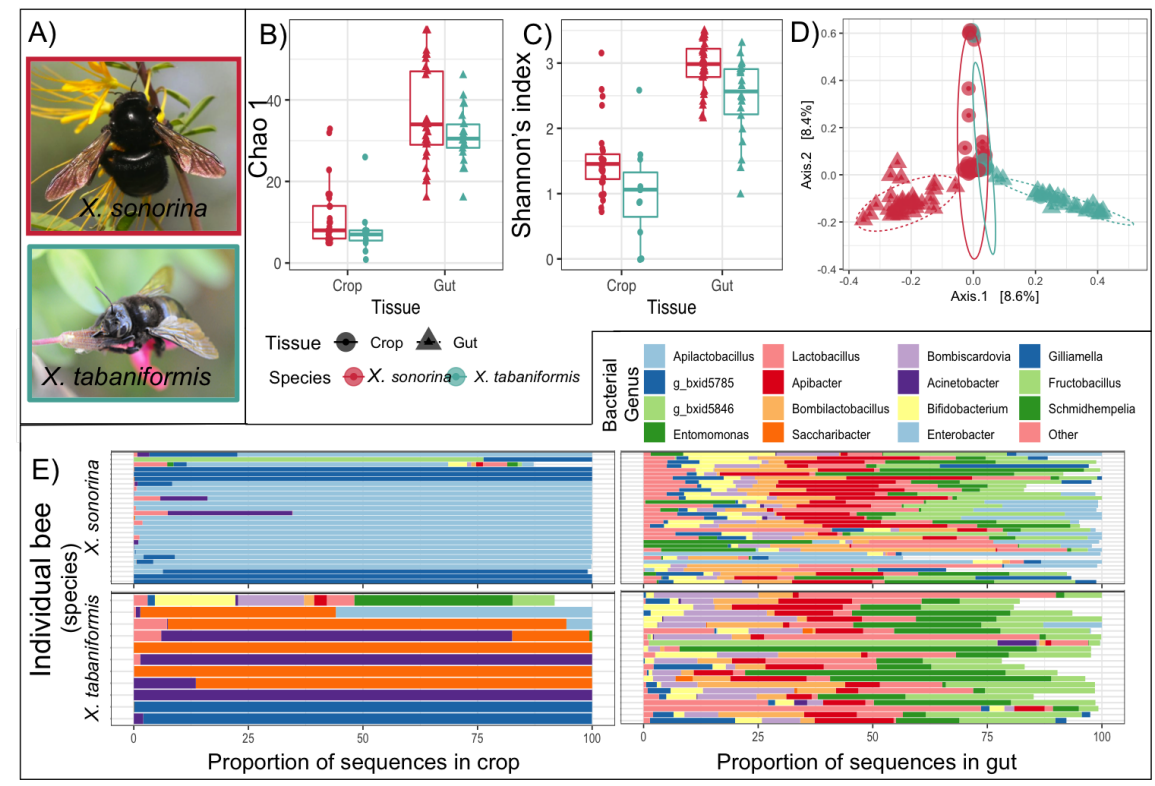




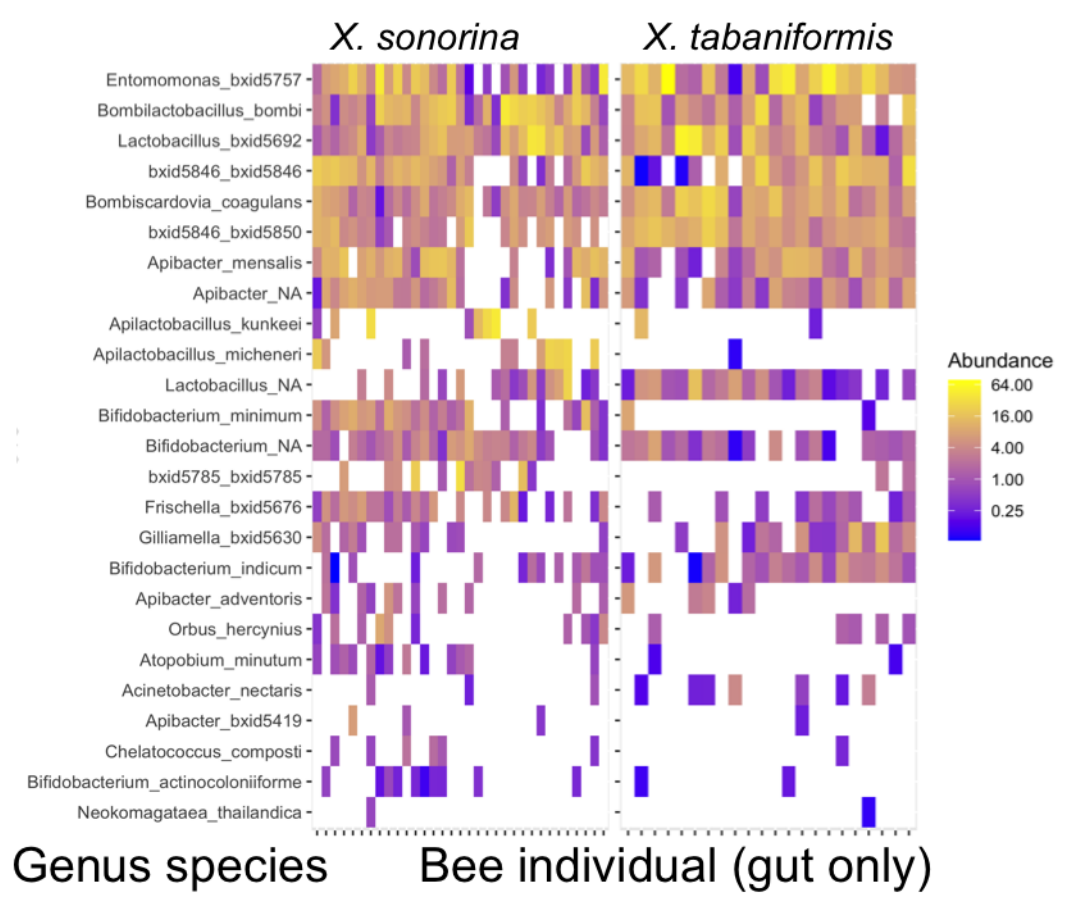

A)

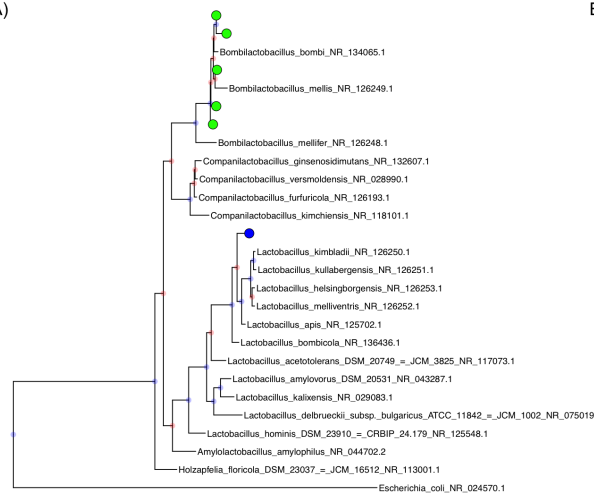

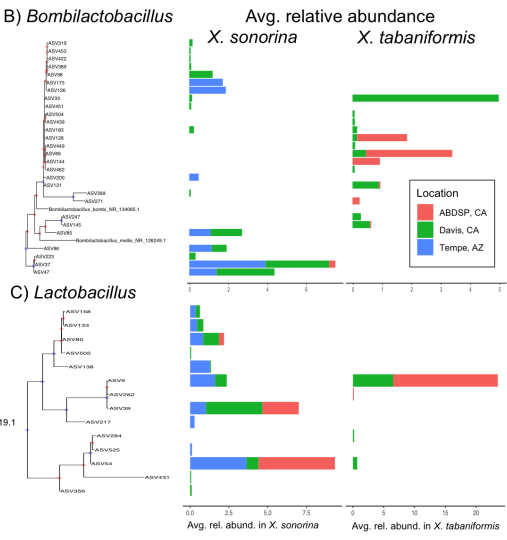



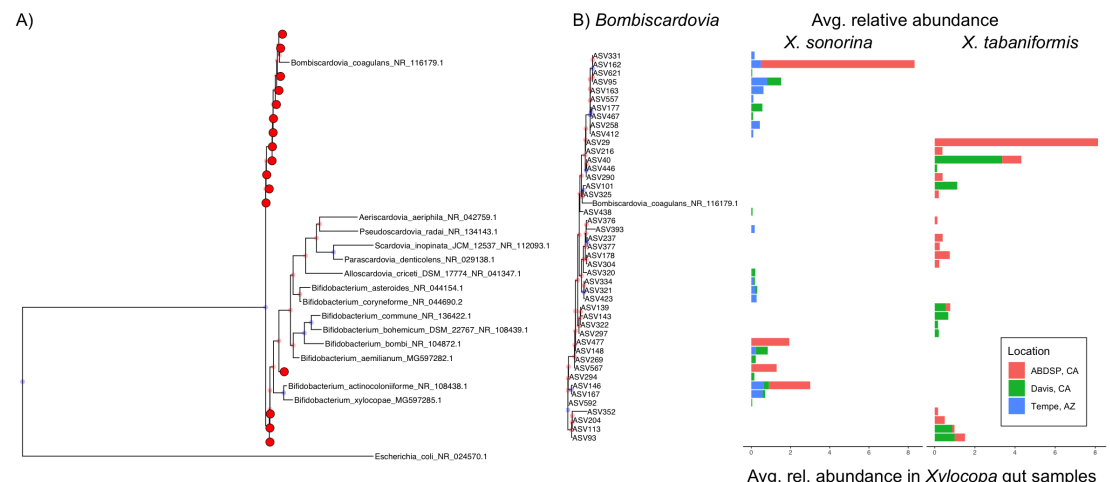